\title{
Imaging Triage of Patients with Late-Window (6-24 Hours) Acute Ischemic Stroke: A Comparative Study Using Multiphase CT Angiography versus CT Perfusion
}

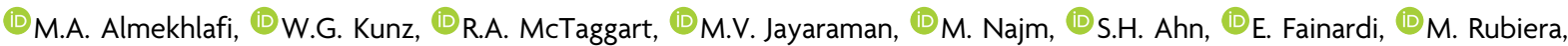

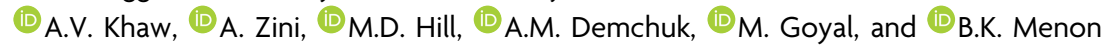

\begin{abstract}
BACKGROUND AND PURPOSE: The role of collateral imaging in selecting patients for endovascular thrombectomy beyond 6 hours from onset has not been established. To assess the comparative utility of collateral imaging using multiphase CTA in selecting late window patients for EVT.
\end{abstract}

MATERIALS AND METHODS: We used data from a prospective multicenter observational study in which all patients underwent imaging with multiphase CT angiography as well as CTP. Two blinded reviewers evaluated patients' eligibility for endovascular thrombectomy using published collateral imaging (multiphase CTA) criteria compared with CTP using the selection criteria of the Clinical Mismatch in the Triage of Wake Up and Late Presenting Strokes Undergoing Neurointervention with Trevo (DAWN) and Endovascular Therapy Following Imaging Evaluation for Ischemic Stroke 3 (DEFUSE-3) trials. CTP images were processed using automated commercial software. The outcomes of patients eligible for endovascular thrombectomy according to multiphase CTA, DAWN, or DEFUSE-3 criteria were compared using multivariable logistic regression modeling. Model characteristics were compared using the C-statistic for the receiver operating characteristic curve, the Akaike information criterion, and the Bayesian information criterion.

RESULTS: Eighty-six patients presented beyond 6 hours from onset/last known well (median, 9.6 hours; interquartile range, 4.1 hours). Thirty-five patients (40.7\%) received endovascular thrombectomy, of whom good functional outcome (90-day mRS, 0-2) was achieved in 16/35 (47\%). Collateral-based imaging paradigms significantly modified the treatment effect of endovascular thrombectomy on 90-day mRS 0-2 ( $\left.P_{\text {interaction }}=.007\right)$. The multiphase CTA-based regression model best fit the data for the 90-day outcome (C-statistic, $0.86 ; 95 \% \mathrm{Cl}, 0.77-0.94$ ) and was associated with the least information loss (Akaike information criterion, 95.7; Bayesian information criterion, 114.9) compared with CTP-based models.

CONCLUSIONS: The collateral-based imaging paradigm using multiphase CTA compares well with CTP in selecting patients for endovascular thrombectomy in the late time window.

ABBREVIATIONS: $\mathrm{AIC}=$ Akaike information criterion; $\mathrm{BIC}=$ Bayesian information criterion; $\mathrm{EVT}=$ endovascular thrombectomy; IQR $=$ interquartile range; $\mathrm{LVO}=$ large-vessel occlusion; $\mathrm{mCTA}=$ multiphase $\mathrm{CTA}$

ndovascular thrombectomy (EVT) has become the standard of care in patients with acute ischemic stroke with large-vessel

Received July 30, 2019; accepted after revision October 1.

From the Department of Clinical Neurosciences (M.A.A., M.N., M.D.H., A.M.D., M.G., B.K.M.), Calgary Stroke Program, Department of Radiology (M.A.A., M.D.H., A.M.D., M.G., B.K.M.), Department of Community Health Sciences (M.A.A., M.D.H., B.K.M.), and Department of Medicine (M.D.H.), Hotchkiss Brain Institute, Cumming School of Medicine University of Calgary, Calgary, Alberta, Canada; Department of Radiology (W.G.K.), University Hospital, Ludwig Maximilian University of Munich, Munich, Germany; Departments of Neurology, Diagnostic Imaging, and Neurosurgery (R.A.M., M.V.J.), Warren Alpert Medical School of Brown University, Providence, Rhode Island; Department of Neurology (S.H.A.), Chosun University School of Medicine, Gwang Ju, South Korea; Department of Neurosciences and Rehabilitation (E.F.), University Hospital, Ferrara, Italy; Department of Neurology (M.R.), Hospital Vall d'Hebron, Passeig de la Vall d'Hebron, Barcelona, Spain; Department of Clinical Neurosciences (A.V.K.), University of Western Ontario, London, Ontario, Canada; and Department of Neurology and Stroke Center (A.Z.), Istituto Di Ricovero e Cura a Carattere Scientifico Istituto di Scienze Neurologiche di Bologna, Maggiore Hospital, Bologna, Italy. occlusion (LVO) presenting within 6 hours from symptom onset. $^{1,2}$ The treatment time window can be further extended to capture patients with salvageable tissue up to 24 hours from last known well. Two randomized trials have shown an overwhelming benefit of EVT over medical treatment in patients presenting up to 16 hours (Endovascular Therapy Following Imaging Evaluation for Ischemic Stroke 3 [DEFUSE-3] $)^{3}$ and 24 hours (Clinical Mismatch in the Triage of Wake Up and Late

Please address correspondence to Bijoy K. Menon, MBBS, MD, DM, MSc, FRCPC Departments of Clinical Neurosciences, Radiology, and Community Health Sciences, Hotchkiss Brain Institute, Cumming School of Medicine, University of Calgary, 1403 29th St NW, Calgary, AB Canada T2N 2T9; e-mail: docbijoymenon@gmail.com; @AlmekhlafiMa; @mayank_G0; @bijoymenon; @HotchkissBrain; @DCNSNeuro; @mobilestroke4U; @MVJayaraman; (WolfgangGKunzMD

http://dx.doi.org/10.3174/ajnr.A6327 
Presenting Strokes Undergoing Neurointervention with Trevo [DAWN]) from last known well. ${ }^{4}$ Both studies used CT perfusion imaging to identify patients with LVO with a limited-size-predicted infarct core or substantial salvageable brain tissue (target mismatch) for inclusion.

The Endovascular Treatment for Small Core and Proximal Occlusion Ischemic Stroke (ESCAPE) trial showed that an imaging paradigm based on measuring collateral status beyond target occlusion is capable of selecting patients with LVO who would benefit from EVT up to 12 hours from onset/last known well. ${ }^{5,6}$ Multiphase CTA (mCTA) is a reliable and well-validated tool for measuring collateral status beyond the target LVO. ${ }^{7-11}$ Collateral assessments on mCTA, even at the brain region level, compare well with CTP-based blood flow measurements in predicting final tissue fate. ${ }^{7}$ Some of the shortcomings of CTP (eg, patient motion, limited brain coverage, larger radiation dose, additional contrast, time, and cost of image postprocessing software) are avoided using mCTA. ${ }^{12,13}$

Recent studies have reported that patient selection using noncontrast CT and single-phase CTA ${ }^{14,15}$ or mCTA in the "late window" after stroke onset is an effective method of selecting patients for EVT. ${ }^{16}$ We compared patient selection using mCTA versus CTP in patients with late-window stroke with LVO using appropriate clinical treatment decisions and 90-day clinical outcomes to assess the comparative utility of each approach.

\section{MATERIALS AND METHODS}

Data are from the Precise and Rapid Assessment of Collaterals Using Multi-Phase CTA in the Triage of Patients with Acute Ischemic Stroke for IV or IA Therapy (PRove-IT) study. ${ }^{7,8,17-19}$ This was a prospective, multicenter international observational study to assess the value of multimodal imaging in the triage of patients with acute ischemic stroke. All patients had noncontrast CT of the brain, CTA of the head and neck, mCTA, and CTP at baseline as described previously. ${ }^{8}$ For this analysis, only patients who presented $\geq 6$ hours from stroke symptom onset/last known well time were included. Images were scored by consensus of 2 readers blinded to all clinical data (except the side of stroke), treatment allocation, and outcome data. In separate sessions and using random patient ordering, the same 2 readers scored the NCCT ASPECTS and then the collateral score on mCTA using a 6-point ordinal pial arterial filling scale as previously described. ${ }^{8}$ CT perfusion images were postprocessed using an automated commercial software. ${ }^{20}$ Symptomatic intracranial hemorrhage was defined using the European Cooperative Acute Stroke Study III (ECASS) III criteria. $^{21}$

For each imaging paradigm, readers assessed whether a patient would be a candidate for EVT (EVT eligibility) based on the following criteria:

1. NCCT and mCTA session: ASPECTS $\geq 5$ and an mCTAbased collateral score of $4-6 .{ }^{8}$

2. DEFUSE-3 trial criteria: predicted core volume of $<70 \mathrm{~mL}$, predicted penumbra volume of $\geq 15 \mathrm{~mL}$, and mismatch ratio of $\geq 1.8 .^{3}$

3. DAWN trial criteria: predicted core volume of $<21 \mathrm{~mL}$ for patients older than 80 years of age $31-51 \mathrm{~mL}$ if younger than 80 years. $^{4}$
We assessed the predictive validity of each imaging paradigm for functional outcomes by comparing logistic regression models generated using data regarding EVT eligibility from each of the above imaging paradigms. For each paradigm, a model was fitted for each of the following outcomes: early neurologic improvement (defined as $\geq 50 \%$ drop in the NIHSS score during 24 hours) and 90-day functional independence (defined as mRS score of 0-2) using logistic regression and ordinal logistic regression with adjustment for key prognostic variables (age, sex, baseline NIHSS score, baseline ASPECTS, onset/last known well to imaging time, and treatment given). Modification of the effect of treatment (EVT versus no EVT) on clinical outcomes by the imaging paradigm was assessed using a multiplicative interaction term. The area under the curve of the receiver operating characteristic properties of the models was compared using the $\chi^{2}$ test as described by Gönen. ${ }^{22}$ In addition, information content of the various models was compared using the Akaike information criterion (AIC) and the Bayesian information criterion (BIC). ${ }^{23}$ These methods aim to identify the model that best approximates the outcome data (ie, the model that minimizes data/information loss) while applying a penalty for model complexity (ie, having too many variables). While the values of AIC or BIC do not have a simple interpretation, the model with the lowest values is the one that is better.

The PRove-IT study was approved by the local ethics review committees. Analyses were performed using STATA 15 software (StataCorp, College Station, Texas).

\section{RESULTS}

\section{Baseline Clinical Data}

Among 614 patients in PRove-IT, 86 patients were included in this analysis as patients with late-window stroke. The median age was 71 years (interquartile range [IQR], 14 years), and $48.8 \%$ were women, while the median baseline NIHSS score was 12 (IQR, 11). The median time from last known well/stroke symptom onset to baseline CT was 9.6 hours (IQR, 4.1 hours). Table 1 summarizes the baseline characteristics of this cohort according to the imaging paradigm and the treatment received.

\section{Baseline Imaging Data}

NCCT and mCTA were available in all patients; CTP, however, could not be processed in 6/86 patients (7\%) due to acquisitionor patient motion-related technical limitations. These 6 patients were assumed to be ineligible for EVT using DAWN and DEFUSE-3 criteria. The median ASPECTS was 9 (IQR, 3). The distribution of the occlusion site was as follows: isolated intracranial ICA (11.6\%), L- or T-type occlusions (12.8\%), M1 MCA (33.7\%), M2 MCA (22.1\%), posterior cerebral artery (1/86, 1.2\%), distal occlusions $(9 / 86,10.5 \%)$, and no occlusions (10/86, 11.6\%). The mean and median baseline infarct volumes were $18.7 \pm$ $28.2 \mathrm{~mL}$ and $8 \mathrm{~mL}$ (range, $0-162 \mathrm{~mL}$ ), and penumbra volumes were $109.5 \pm 10.7 \mathrm{~mL}$ and $94 \mathrm{~mL}$ (range, $0-753 \mathrm{~mL}$ ), respectively.

\section{Treatment and Outcome Data}

Of 86 patients, 35 patients (40.7\%) received EVT, while $51(59.3 \%)$ were treated conservatively. Among EVT-treated patients, successful reperfusion (modified TICI 2b/3) was 


\begin{tabular}{|c|c|c|c|c|c|c|}
\hline & \multicolumn{6}{|c|}{ PRove-IT Late-Window Cohort $(n=86)$} \\
\hline & \multicolumn{2}{|c|}{ Favorable mCTA Profile $(n=63)$} & \multicolumn{2}{|c|}{ Favorable DEFUSE Profile $(n=58)$} & \multicolumn{2}{|c|}{ Favorable DAWN Profile $(n=32)$} \\
\hline & EVT & No EVT & EVT & No EVT & EVT & No EVT \\
\hline No. & $33(52.4 \%)$ & $30(47.6 \%)$ & $28(48.3 \%)$ & $30(51.7 \%)$ & $18(56.3 \%)$ & $14(43.7 \%)$ \\
\hline $\mathrm{Age}^{\mathrm{a}}$ & 75 & 72 & 75 & 72 & 75 & 77 \\
\hline NIHSS score ${ }^{a}$ & 18 & 12 & 18 & 14 & 19 & 18 \\
\hline ASPECTS $^{\mathrm{a}}$ & 9 & 9 & 9 & 8 & 9 & 9 \\
\hline $\begin{array}{l}\text { Onset/LSN to } \\
\text { imaging (min) }\end{array}$ & 578 (9.6 hr) & 580 (9.7 hr) & 559 (9.3 hr) & 571 (9.5 hr) & 608 (10.1 hr) & 593 (9.9 hr) \\
\hline \multicolumn{7}{|l|}{ Occlusion site } \\
\hline Terminal ICA & $12(36.4 \%)$ & $3(10 \%)$ & $10(35.7 \%)$ & $4(13.3 \%)$ & $7(38.9 \%)$ & $3(21.4 \%)$ \\
\hline M1 & $14(42.4 \%)$ & $13(43.3 \%)$ & 10 (35.7\%) & 13 (43.3\%) & $6(33.3 \%)$ & 5 (35.7\%) \\
\hline M2 & $6(18.2 \%)$ & 14 (46.7\%) & $7(25 \%)$ & $13(43.3 \%)$ & $4(22.2 \%)$ & $6(42.9 \%)$ \\
\hline M3 or distal & $1(3 \%)$ & 0 & $1(3.6 \%)$ & 0 & $1(5.6 \%)$ & 0 \\
\hline
\end{tabular}

Note:-LSN indicates last seen normal.

${ }^{\mathrm{a}}$ Denotes median.

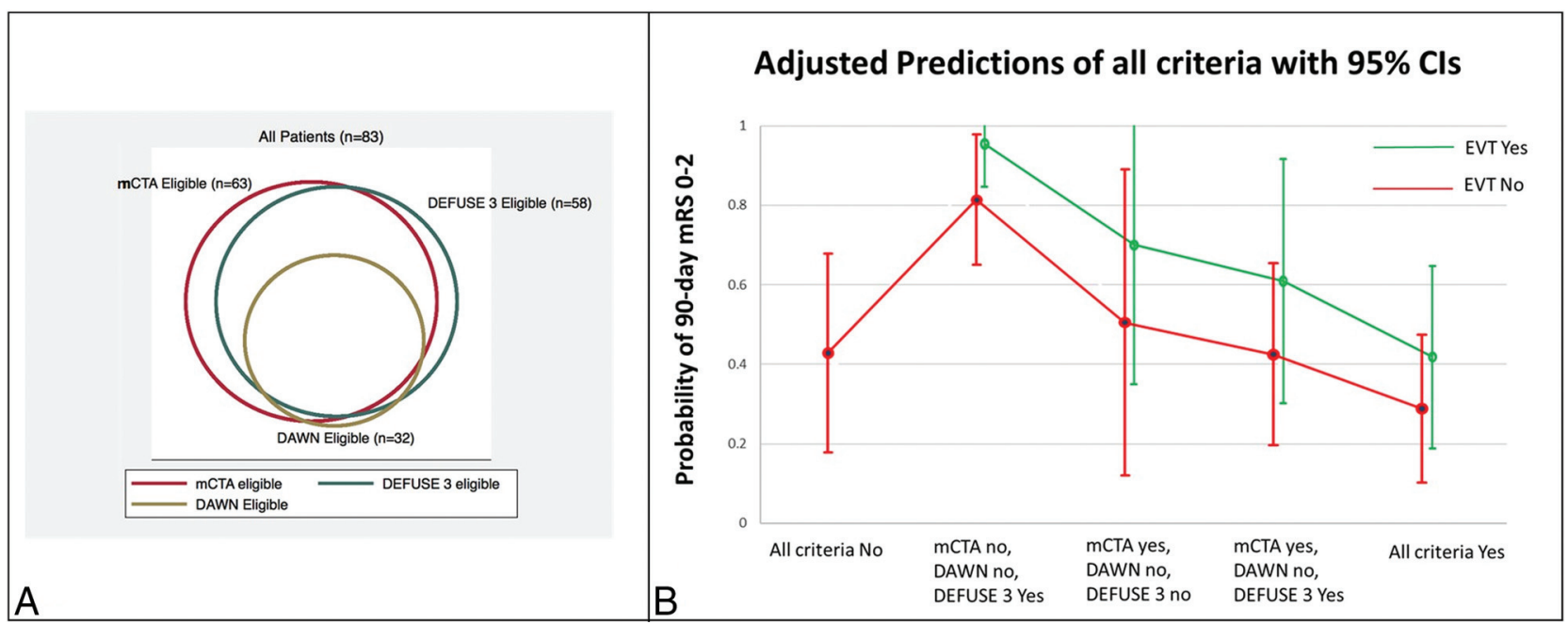

FIG 1. A, Proportion of patients eligible for EVT according to each imaging criterion. $B$, Adjusted predicted probability of achieving 90 -day mRS score of 0-2 in those meeting none, some, or all the imaging criteria when treated with EVT (green) versus not (red).

achieved in $71.4 \%(25 / 35)$ patients. The median 24-hour NIHSS score in the overall cohort was 7 (IQR, 13). Early neurologic improvement was seen in $27 / 86$ patients $(31.4 \%)$ in the cohort overall and in $54.3 \%(19 / 35)$ of those treated with EVT $(72 \%$; $18 / 25$ of those with successful reperfusion). Four of 86 patients (4.7\%) had symptomatic intracranial hemorrhage; all received EVT (4/35, 11.4\% of EVT-treated patients). Of the patients with symptomatic intracranial hemorrhage, all (4/4) met the DEFUSE-3 criteria for EVT, while $3 / 4$ met the mCTA and DAWN criteria for EVT eligibility. Functional outcome data at 90 days were available for 83 of 86 patients (96.5\%). Independent outcome (mRS, 0-2) was achieved in 47\% (39/ 83 ) in the overall cohort and in $51.4 \%(18 / 35)$ of those treated with $\operatorname{EVT}(72 \%, 18 / 25$ of those with successful reperfusion).

\section{Eligibility for EVT and Predictive Validity of Imaging Paradigms}

Of the 83 patients in this cohort with available 90 -day outcomes, 63 patients (75.9\%) were considered eligible for EVT according to the mCTA criteria, compared with 58 patients $(69.9 \%)$ according to the DEFUSE- 3 criteria and only 32 patients (38.6\%) according to DAWN criteria (Fig $1 A$ ). Among the 35 EVTtreated patients in our cohort, 33 patients $(94.3 \%)$ were considered good EVT candidates according to the MCTA criteria compared with 28 patients (80\%), according to DEFUSE- 3 criteria, and only 18 patients (51.4\%), according to DAWN criteria for EVT eligibility. Among EVT-treated patients, the proportion of patients with the highest early neurologic improvement and independent 90 -day functional outcome were those who were good treatment candidates according to mCTA paradigm. Similarly, patients who were not EVT candidates according to mCTA paradigm had the lowest likelihood of having early neurologic improvement and an independent 90-day functional outcome.

The predictive validity of each imaging paradigm in determining prespecified clinical outcomes was assessed using logistic regression models (Table 2 and Fig $1 B$ ). All imaging paradigms performed well in predicting 90-day functional outcome versus early neurologic improvement. Patients who had a favorable mCTA imaging profile had significantly better 90 -day functional 
Table 2: Comparison of the ability of imaging paradigms in discriminating clinical outcomes using logistic regression modeling ${ }^{\mathrm{a}}$

\begin{tabular}{|c|c|c|c|c|c|c|}
\hline \multicolumn{7}{|c|}{ Comparison } \\
\hline Imaging Paradigm/Criteria & No. & Odds Ratio $(95 \% \mathrm{Cl})$ & $P$ Value & C-Statistic & $\mathrm{AIC} / \mathrm{BIC}$ & $\mathrm{AIC/BIC} \mathrm{(0)}$ \\
\hline \multicolumn{7}{|l|}{ 90-day mRS } \\
\hline mCTA (>3 vs $\leq 3$ ) & 82 & $9.6(1.9-48.8)$ & .001 & 0.86 & $95.7 / 114.9$ & $300.6 / 331.9$ \\
\hline DEFUSE-3 criteria & 82 & $5.5(1.2-25.3)$ & .028 & 0.84 & $99.0 / 118.3$ & \\
\hline DAWN criteria & 82 & $9.3(0.9-98.8)$ & .065 & 0.83 & $99.3 / 118.6$ & $303.1 / 334.4$ \\
\hline \multicolumn{7}{|c|}{$\begin{array}{l}\text { Early neurologic improvement ( } \geq 50 \% \text { drop } \\
\text { in } 24-\mathrm{hr} \text { NIHSS score from baseline) }\end{array}$} \\
\hline $\operatorname{mCTA}(>3$ vs $\leq 3)$ & 82 & $13.3(2.9-61)$ & .001 & 0.80 & 98.2 & 117.5 \\
\hline DEFUSE-3 criteria & 82 & $8.5(1.9-37.5)$ & .005 & 0.74 & 105.9 & 125.1 \\
\hline DAWN criteria & 82 & $5.6(0.6-56.1)$ & .141 & 0.71 & 109.6 & \\
\hline
\end{tabular}

${ }^{a}$ Variables age, sex, baseline NIHSS score, baseline NCCT ASPECTS, onset/last known well to imaging time, EVT, and the interaction term imaging paradigm $\times$ EVT (yes versus no) were included in all models. C-statistic represents the area under a receiver operating characteristic curve. AIC and BIC are Bayesian information criteria methods to assess model fit in which the model with the lowest $\mathrm{AIC}$ or $\mathrm{BIC}$ is preferred. $\mathrm{AIC} / \mathrm{BIC}(\mathrm{O})$ denotes the $\mathrm{AIC}$ and $\mathrm{BIC}$ for the ordinal regression models.

outcome when they were treated with EVT (mCTA/EVT, $\left.P_{\text {interaction }}=.007\right)$. This was also noted for the paradigm based on DEFUSE-3 criteria $\left(P_{\text {interaction }}=.028\right)$ but not for the DAWN criteria $\left(P_{\text {interaction }}=.065\right)$. The probability of 90 -day independent functional outcome was highest among patients meeting DEFUSE-3 criteria both with and without EVT (Fig 1B).

For both outcomes, models using mCTA were not significantly better than the next-best model for clinical outcomes when comparing the C-statistic ( $\chi^{2}$ test for model comparisons, $P>$.05). For both outcomes however, models using the mCTA criteria minimized information loss (AIC and BIC values) better than either of the models that used CTP. For the 90-day mRS, after the mCTA model, the next-best model was the DEFUSE-3based perfusion criteria ( 0.19 times as probable as the mCTAbased model to minimize information loss). For both outcomes, the next-best model was the perfusion imaging paradigm using the DEFUSE-3 criteria. Similarly, when using ordinal logistic regression for 90-day outcome, the model using the mCTA criteria best minimized information loss (lowest AIC and BIC values) compared with models that used CTP.

\section{DISCUSSION}

This analysis shows that collateral-based imaging paradigms using mCTA are at least as good as perfusion-based imaging paradigms in selecting patients presenting late for EVT. Models using mCTA had the highest likelihood of discriminating those who would have good clinical outcome when treated with EVT versus those who would not. Models using DEFUSE-3 selection criteria had better predictive validity in determining good clinical outcomes among EVT-treated patients than the DAWN paradigm. Models using mCTA or DEFUSE-3 criteria may result in more patients being offered EVT (Fig 1A).

In patients presenting within 6 hours from symptom onset/ last known well, current guidelines recommend the use of NCCT and CTA in determining patient eligibility for EVT. The primary goal of imaging selection in these patients is to identify a patient with an ASPECTS of $\geq 6$ and a proximal intracranial occlusion. ${ }^{1}$ In patients presenting beyond 6 hours, current guidelines suggest identifying LVO using CTA and the DAWN or DEFUSE-3 eligibility criteria for patient selection. Most interesting, the American Heart Association guidelines suggest that the benefit with EVT was independently demonstrated for both the subgroup of patients who met DAWN eligibility criteria and for the subgroup who did not. ${ }^{1}$ This is important because the findings of DAWN and DEFUSE-3 trials do not role out EVT befits in patients who do not fit their imaging selection criteria.

Although perfusion imaging variability and interpretation have benefited from the use of automated software, challenges related to perfusion imaging such as time delays with acquisition, training of technologists, motion sensitivity, and the risks with extra radiation and contrast persist. In this analysis, $7 \%$ of patients' perfusion images could not be analyzed by postprocessing software. Moreover, a recent study suggests that perfusionbased selection criteria disqualified more patients from EVT compared with other selection paradigms, without improving outcomes. ${ }^{24}$ While our analyses showed that patients meeting the DEFUSE-3 criteria had the highest predicted probability of functional independence, those patients did equally well with and without EVT. The treatment effect (ie, the difference in the proportion of good outcome when treated with EVT versus not having it) was higher in patients selected using the mCTA criteria alone rather than with other criteria (Fig $1 B$ ).

NCCT and CTA continue to be the workhorses of acute stroke diagnosis. Thus, using these modalities for determining eligibility, even in the extended time window, is a logical next step. Recent studies have shown that stroke centers that use an NCCT- and mCTA-based imaging paradigm to select patients for EVT in the extended time window achieve results that are comparable with those obtained in patients who received EVT in the DAWN and DEFUSE-3 trials. ${ }^{14-16}$ A post hoc analysis of the ESCAPE trial restricted to patients who presented late showed that patients receiving EVT had similar prospects of good clinical outcome (48.5\%) versus those in the DAWN (49\%) and DEFUSE-3 (45\%) trials. ${ }^{6}$ Taken together, this analysis and other previous analyses provide more evidence to support the construct that an mCTA-based imaging paradigm is at least as good as current perfusion-based imaging paradigms in selecting patients presenting late for EVT. ${ }^{8}$ The adoption of NCCT-/mCTA-based imaging selection for the 6- to 24-hour time window would also have implications for stroke systems of care because this simplified imaging paradigm can be used at Primary Stroke Centers to triage patients for EVT without the need for implementing perfusion imaging. ${ }^{13,25}$

Our study has limitations. First, a nonrandomized study of this nature is vulnerable to selection bias. Our inclusion of 
patients who did not receive EVT is an attempt to mitigate any effects of bias related to selecting patients for EVT based on nonimaging factors (eg, age or stroke symptom severity). Second, this analysis did not differentiate between patients who had wake-up strokes from those with a witnessed onset that was 6 hours prior. Third, the images were read by 2 expert readers and not in real life. Automating collateral assessments and even NCCT ASPECTS interpretation using validated algorithms could be potential solutions to address this latter concern.

\section{CONCLUSIONS}

In this prospective study of patients presenting after 6 hours from onset/time last known well, an mCTA collateral-based paradigm is at least as good as guideline-approved perfusion imaging-based paradigms in selecting patients for EVT.

Disclosures: Mohammed Almekhlafi-UNRELATED: Employment: University of Calgary; Grants/Grants Pending: Canadian Institutes of Health Research.* Alexander V. Khaw-UNRELATED: Grants/Grants Pending: Lawson Health Research Institute, Comments: grant for investigator-initiated study.* Andrea ZiniUNRELATED: Board Membership: Stryker, Boehringer Ingelheim; Consultancy: Medtronic, Boehringer Ingelheim; Payment for Lectures Including Service on Speakers Bureaus: Medtronic, Cerenovus*; Travel/Accommodations/Meeting Expenses Unrelated to Activities Listed: Medtronic, Boehringer Ingelheim, Stryker.* Michael D. Hill—UNRELATED: Consultancy: Boehringer Ingelheim, Comments: COLUMBUS registry, involvement completed June 2019; Grants/Grants Pending: NoNO Inc, Medtronic, Stryker, Comments: ESCAPE-NAl trial, HERMES collaboration, UNMASK EVT study.* Andrew Demchuk—RELATED: Grant: CIHR*; Consulting fee or honorarium: Medtronic; UNRELATED: Employment. University of Calgary; Patents (planned, pending or issued): Circle NVI, Comments: Stroke imaging software. Mayank Goyal—UNRELATED: Consultancy: Medtronic, Stryker, Mentice, MicroVention, Comments: advice regarding acute stroke devices and procedures; Grants/ Grants Pending: Stryker, Comments: unrestricted grant to University of Calgary concerning the UNMASK EVT study*; Other: GE Healthcare, Comments: licensing agreement related to Systems of Acute Stroke Diagnosis. Bijoy K. Menon—UNRELATED: Grant: PRovelT Canadian Institutes of Health Research.* *Money paid to the institution.

\section{REFERENCES}

1. Powers WJ, Rabinstein AA, Ackerson T, et al. 2018 Guidelines for the Early Management of Patients with Acute Ischemic Stroke: A Guideline for Healthcare Professionals from the American Heart Association/American Stroke Association. Stroke 2018;49:e46-110 CrossRef Medline

2. Goyal M, Menon BK, van Zwam WH, et al. Endovascular thrombectomy after large-vessel ischaemic stroke: a meta-analysis of individual patient data from five randomised trials. Lancet 2016;387:1723-31 CrossRef Medline

3. Albers GW, Marks MP, Kemp S, et al. Thrombectomy for stroke at 6 to 16 hours with selection by perfusion imaging. $N$ Engl J Med 2018;378:708-18 CrossRef Medline

4. Nogueira RG, Jadhav AP, Haussen DC, et al. Thrombectomy 6 to 24 hours after stroke with a mismatch between deficit and infarct. $N$ Engl J Med 2018;378:11-21 CrossRef Medline

5. Goyal M, Demchuk AM, Menon BK, et al. Randomized assessment of rapid endovascular treatment of ischemic stroke. $N$ Engl J Med 2015;372:1019-30 CrossRef Medline

6. Evans JW, Graham BR, Pordeli P, et al. Time for a time window extension: insights from late presenters in the ESCAPE Trial. AJNR Am J Neuroradiol 2018;39:102-06 CrossRef Medline

7. d'Esterre CD, Trivedi A, Pordeli P, et al. Regional comparison of multiphase computed tomographic angiography and computed tomographic perfusion for prediction of tissue fate in ischemic stroke. Stroke 2017;48:939-45 CrossRef Medline

8. Menon BK, d'Esterre CD, Qazi EM, et al. Multiphase CT angiography: a new tool for the imaging triage of patients with acute ischemic stroke. Radiology 2015;275:510-20 CrossRef Medline

9. García-Tornel A, Carvalho V, Boned S, et al. Improving the evaluation of collateral circulation by multiphase computed tomography angiography in acute stroke patients treated with endovascular reperfusion therapies. Interv Neurol 2016;5:209-17 CrossRef Medline

10. Di Giuliano F, Picchi E, Sallustio F, et al. Accuracy of advanced CT imaging in prediction of functional outcome after endovascular treatment in patients with large-vessel occlusion. Neuroradiol J 2019;32:62-70 CrossRef Medline

11. Uransilp N, Dharmasaroja PA, Watcharakorn A, et al. Implementation of multiphase computed tomography angiography in management of patients with acute ischemic stroke in clinical practice. J Clin Neurosci 2019;62:100-04 CrossRef Medline

12. Goyal M, Menon BK, Derdeyn CP. Perfusion imaging in acute ischemic stroke: let us improve the science before changing clinical practice. Radiology 2013;266:16-21 CrossRef Medline

13. Almekhlafi MA, Kunz WG, Menon BK, et al. Imaging of patients with suspected large-vessel occlusion at primary stroke centers: available modalities and a suggested approach. AJNR Am J Neuroradiol 2019;40:396-400 CrossRef Medline

14. Santos T, Carvalho A, Cunha AA, et al. NCCT and CTA-based imaging protocol for endovascular treatment selection in late presenting or wake-up strokes. J Neurointerv Surg 2019;11:200-03 CrossRef Medline

15. DiBiasio E, Jayaraman M, Goyal M, et al. Dismantling the ability of CT and MRI to identify the target mismatch profile in patients with anterior circulation large vessel occlusion beyond six hours from symptom onset. Emerg Radiol 2019;26:401-08 CrossRef Medline

16. Motyer R, Thornton J, Power S, et al. Endovascular thrombectomy beyond 12 hours of stroke onset: a stroke network's experience of late intervention. J Neurointerv Surg 2018;10:1043-46 CrossRef Medline

17. Casault C, Al Sultan AS, Trivedi A, et al. Collateral scoring on CT angiogram must evaluate phase and regional pattern. Can J Neurol Sci 2017;44:503-07 CrossRef Medline

18. d'Esterre CD, Boesen ME, Ahn SH, et al. Time-dependent computed tomographic perfusion thresholds for patients with acute ischemic stroke. Stroke 2015;46:3390-97 CrossRef Medline

19. Ahn SH, d'Esterre CD, Qazi EM, et al. Occult anterograde flow is an under-recognized but crucial predictor of early recanalization with intravenous tissue-type plasminogen activator. Stroke 2015;46:96875 CrossRef Medline

20. Campbell BC, Yassi N, Ma H, et al. Imaging selection in ischemic stroke: feasibility of automated CT-perfusion analysis. Int J Stroke 2015;10:51-54 CrossRef Medline

21. Hacke W, Kaste M, Bluhmki E, et al. Thrombolysis with alteplase 3 to 4.5 hours after acute ischemic stroke. $N$ Engl J Med 2008; 359:1317-29 CrossRef Medline

22. Gönen M. Analyzing Receiver Operating Characteristic Curves with SAS. SAS Institute, 2007

23. Burnham KP, Anderson DR. Multimodel inference: understanding AIC and BIC in model selection. Sociol Methods Res 2004;33:261304 CrossRef

24. Bouslama M, Bowen MT, Haussen DC, et al. Selection paradigms for large vessel occlusion acute ischemic stroke endovascular therapy. Cerebrovasc Dis 2017;44:277-84 CrossRef Medline

25. McTaggart RA, Yaghi S, Cutting SM, et al. Association of a primary stroke center protocol for suspected stroke by large-vessel occlusion with efficiency of care and patient outcomes. JAMA Neurol 2017;74:793-800 CrossRef Medline 